Encyclopedia of Feeding and Eating

Disorders 

Tracey Wade

Editor

\section{Encyclopedia of Feeding and Eating Disorders}

With 64 Figures and 50 Tables

第 Springer 
Editor

Tracey Wade

School of Psychology

Flinders University

Adelaide, SA, Australia

ISBN 978-981-287-103-9

ISBN 978-981-287-104-6 (eBook)

ISBN 978-981-287-105-3 (print and electronic bundle)

DOI 10.1007/978-981-287-104-6

Library of Congress Control Number: 2017937657

(C) Springer Nature Singapore Pte Ltd. 2017

This work is subject to copyright. All rights are reserved by the Publisher, whether the whole or part of the material is concerned, specifically the rights of translation, reprinting, reuse of illustrations, recitation, broadcasting, reproduction on microfilms or in any other physical way, and transmission or information storage and retrieval, electronic adaptation, computer software, or by similar or dissimilar methodology now known or hereafter developed.

The use of general descriptive names, registered names, trademarks, service marks, etc. in this publication does not imply, even in the absence of a specific statement, that such names are exempt from the relevant protective laws and regulations and therefore free for general use.

The publisher, the authors and the editors are safe to assume that the advice and information in this book are believed to be true and accurate at the date of publication. Neither the publisher nor the authors or the editors give a warranty, express or implied, with respect to the material contained herein or for any errors or omissions that may have been made. The publisher remains neutral with regard to jurisdictional claims in published maps and institutional affiliations.

Printed on acid-free paper

This Springer imprint is published by Springer Nature

The registered company Springer Nature Singapore Pte Ltd.

The registered company address is: 152 Beach Road, \#21-01/04 Gateway East, Singapore 189721, Singapore 
To all those colleagues, reviewers, students, and clients who have inspired me to try to make a meaningful contribution to our understanding of feeding and eating disorders 



\section{Preface}

This scholarly Encyclopedia of Feeding and Eating Disorders aims to present a comprehensive overview of the field by a variety of experts currently working and researching in this area. Given the changes to this diagnostic category introduced in the Diagnostic and Statistical Manual of Mental Disorders, Fifth Edition (DSM-5) in 2013, it seems timely to produce such a resource. The encyclopedia and associated web-based material covers feeding and eating disorders from "bench to bedside," with respect to genetics, biology, and neuroscience through to theory and its application in developing clinical approaches to the prevention and treatment of feeding and eating disorders.

In order for this work to become a reality, it required hard and dedicated work from a strong editorial team who are recognized as leaders in the field of eating disorders in order to commission the breadth of quality contributions that were necessary for such a work. This team included the following people: Karina Allen from the University of Western Australia (comorbidities), Kelly Berg from the University of Minnesota (assessment), Michael Kohn from the University of Sydney (medical issues), Glenn Waller (University of Sheffield) and Ulrike Schmidt (King's College London) working together on the evidence-based treatment section, Jennifer Wildes from the University of Pittsburgh (classification and epidemiology), and Simon Wilksch from Flinders University in South Australia (prevention).

Such a scholarly work would not be possible without the contribution of many different authors across many different countries, generously sharing their expert summary of the current state of play of our understanding of the issues that impact on feeding and eating disorders and how to work with these disorders. It is my hope that by bringing together such a diverse and learned range of contributions that we can move closer to better preventing and treating feeding and eating disorders in such a way that minimizes the negative impact that they can have on so many lives.

The encyclopedia will have an important impact on a large audience as the material covers basic through to more advanced topics. It will not only be particularly useful to individuals early in their careers of research and clinical training, but also to more advanced professionals who are interested in feeding and eating disorders as part of a range of research or clinical interests, as it provides a practical "on hand" perspective for such individuals.

April 2017

Tracey Wade Editor 



\section{About the Editor}

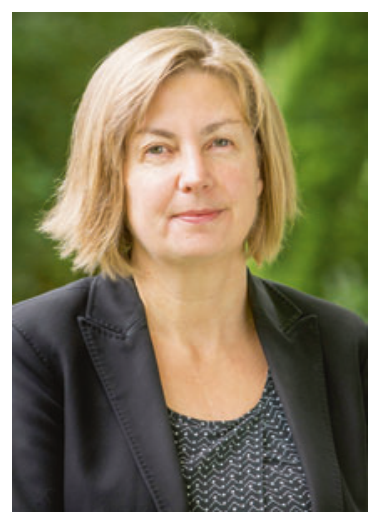

Tracey Wade completed a Masters of Clinical Psychology at the Australian National University in 1992, a Ph.D. at Flinders University in 1998, and a post doctoral fellowship in the USA during 1999 at the Virginia Institute for Psychiatric and Behavioral Genetics under the supervision of Professors Cynthia Bulik and Kenneth Kendler. Since 2000, she has worked in the School of Psychology at Flinders University, South Australia, where she currently holds a position as a Professor. She was awarded the Australian Psychological Society (APS) Ian M Campbell Memorial Prize for outstanding contribution to the scientific or professional status of Clinical Psychology in Australia in 2000, and in 2003 she was awarded the APS Early Career Award. She served as Editor-in-Chief of the APS journal Clinical Psychologist from 2010 to 2014. From 2017 to 2021, she will serve as Associate Editor for the International Journal of Eating Disorders.

Dr. Wade has worked as a clinician in the area of eating disorders for almost 30 years. She is on the management committee responsible for the oversight and development of the new Statewide Eating Disorders Service in South Australia and is a member of the Steering Committee of the National Eating Disorder Collaboration. Her current research interests are in the etiology (including genetic epidemiology), prevention, and treatment of eating disorders and perfectionism. She has cowritten 2 books on the treatment of perfectionism and has over 160 publications in peer-reviewed journals. In 2015, she 
was elected a Fellow of the Academy of the Social Sciences in Australia, in 2016 she was made an Inaugural Honorary Fellow of the Australian Association for Cognitive and Behaviour Therapy, and in 2018 she will be the President of the Eating Disorder Research Society. 


\section{Section Editors}

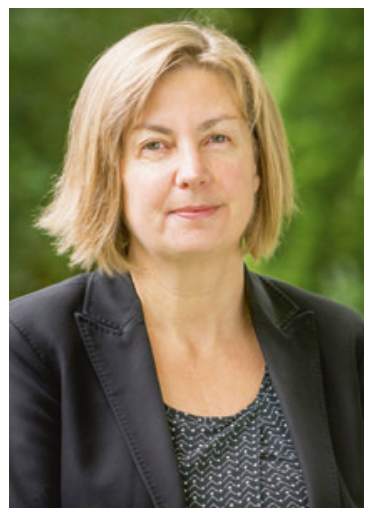

Tracey Wade School of Psychology, Flinders University, Adelaide, SA, Australia

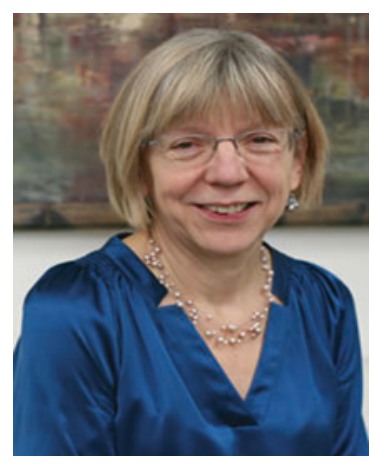

Ulrike Schmidt Institute of Psychiatry, Psychology and Neuroscience, King's College London, London, UK 


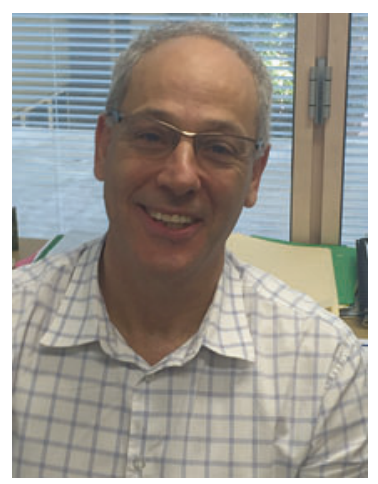

Michael Kohn Sydney University School of Medicine, Adolescent and Young Adult Services Western Sydney, Centre for Research into Adolescent's Health, Adolescent and Young Adult Medicine, Westmead Hospital, Westmead, Sydney, NSW, Australia

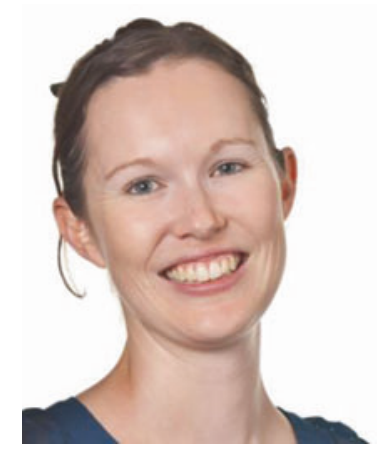

Karina Allen The Eating Disorders Service, Maudsley Hospital, South London and Maudsley NHS Foundation Trust, London, UK

Institute of Psychiatry, Psychology and Neuroscience, King's College London, London, UK

School of Psychology, The University of Western Australia, Crawley, WA, Australia

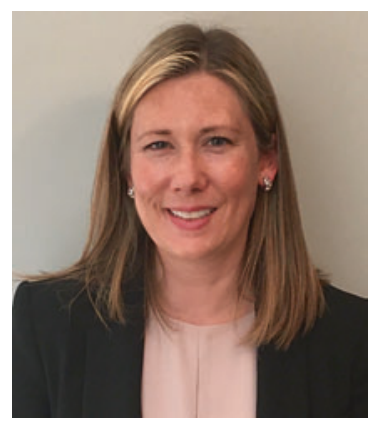

Jennifer E. Wildes Eating Disorders Program, Department of Psychiatry and Behavioral Neuroscience, The University of Chicago, Chicago, IL, USA 


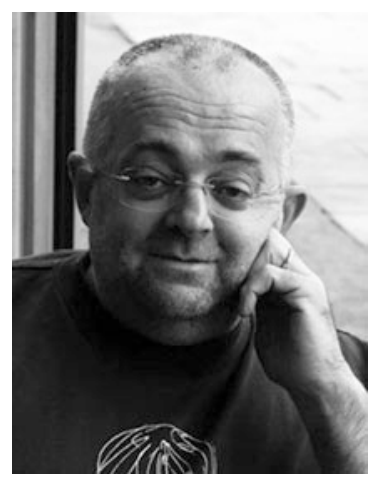

Glenn Waller Clinical Psychology Unit, Department of Psychology, The University of Sheffield, Sheffield, South Yorkshire, UK

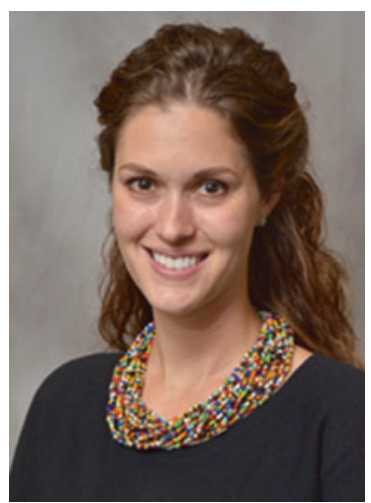

Kelly C. Berg Department of Psychiatry, The University of Minnesota, Minneapolis, MN, USA

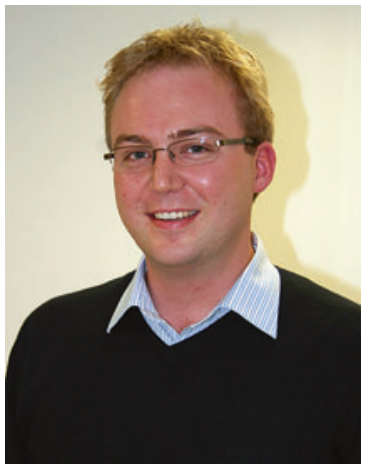

Simon Wilksch School of Psychology, Flinders University, Adelaide, SA, Australia 



\section{Contributors}

Erin C. Accurso Department of Psychiatry, University of California, San Francisco, San Francisco, CA, USA

Karina Allen The Eating Disorders Service, Maudsley Hospital, South London and Maudsley NHS Foundation Trust, London, UK

Institute of Psychiatry, Psychology and Neuroscience, King's College London, London, UK

School of Psychology, The University of Western Australia, Crawley, WA, Australia

Drew Anderson College of Arts and Sciences, Department of Psychology, University at Albany, State University of New York, Albany, NY, USA

Gail Anderson Department of Adolescent Medicine, Westmead Hospital, Westmead, NSW, Australia

Lisa M. Anderson College of Arts and Sciences, Department of Psychology, University at Albany, State University of New York, Albany, NY, USA

Julia Antonini Ontario Community Outreach Program for Eating Disorders, University Health Network, Toronto, ON, Canada

Katherine D. Arnow Stanford University, Palo Alto, CA, USA

Melissa J. Atkinson Department of Psychology, Centre for Appearance Research, University of the West of England, Bristol, UK

Evelyn Attia Department of Psychiatry, Columbia University Medical Center, Weill Cornell Medical College, New York, NY, USA

Molly E. Atwood Department of Psychology, Ryerson University, Toronto, ON, Canada

Jessica H. Baker Department of Psychiatry, University of North Carolina at Chapel Hill, Chapel Hill, NC, USA

Marissa Barmine Department of Medical and Clinical Psychology, Uniformed Services University of the Health Sciences (USUHS), Bethesda, MD, USA

Carolyn Black Becker Department of Psychology, Trinity University, San Antonio, TX, USA 
Ina Beintner Department of Clinical Psychology and Psychotherapy, Technical University Dresden, Dresden, Germany

Kelly C. Berg Department of Psychiatry, The University of Minnesota, Minneapolis, MN, USA

Jennifer Bleck East Stroudsburg University, East Stroudsburg, PA, USA

Kerstin K. Blomquist Furman University, Greenville, SC, USA

Leah Boepple Department of Psychology, University of South Florida, Tampa, FL, USA

Mary M. Boggiano Department of Psychology, University of Alabama at Birmingham, College of Arts and Sciences, Birmingham, AL, USA

Kristin Bohn Department of Psychiatry, Waneford Hospital, University of Oxford, Centre for Research on Dissemination at Oxford (CREDO), Oxford, UK

Cara Bohon Stanford University School of Medicine, Stanford, CA, USA

Brittany K. Bohrer Department of Psychology, University of Kansas, Lawrence, KS, USA

Angeline Bottera Department of Psychology, Trinity University, San Antonio, TX, USA

Tosca D. Braun Department of Psychology, University of Connecticut, Storrs, CT, USA

Amanda J. Brown Department of Psychiatry, Columbia University College of Physicians and Surgeons, New York, NY, USA

Division of Clinical Therapeutics, New York State Psychiatric Institute, New York, NY, USA

Amy Brown Eating Disorders Unit, South London and Maudsley NHS Foundation Trust, London, UK

Cynthia M. Bulik Departments of Psychiatry and Nutrition, University of North Carolina at Chapel Hill, Chapel Hill, NC, USA

Department of Medical Epidemiology and Biostatistics, Karolinska Institutet, Stockholm, Sweden

Emily Bulik-Sullivan Department of Medicine, Center for Gastrointestinal Biology and Disease, University of North Carolina at Chapel Hill, Chapel Hill, $\mathrm{NC}$, USA

Emilee E. Burgess Department of Psychology, University of Alabama at Birmingham, College of Arts and Sciences, Birmingham, AL, USA

Meghan E. Byrne Washington University in St. Louis, St. Louis, CA, USA

Susan Byrne School of Psychology, University of Western Australia, Perth, Crawley, WA, Australia

Centre for Clinical Interventions, Perth, WA, Australia 
Terry Carney Faculty of Law Building, Sydney Law School, University of Sydney, Sydney, NSW, Australia

Ian Carroll Departments of Nutrition, University of North Carolina at Chapel Hill, Chapel Hill, NC, USA

Jacqueline C. Carter Department of Psychology, Memorial University of Newfoundland, St. John's, NF, Canada

Thomas F. Cash Old Dominion University, Norfolk, VA, USA

Stephanie E. Cassin Department of Psychology, Ryerson University, Toronto, ON, Canada

Department of Psychiatry, University of Toronto, Toronto, ON, Canada

Centre for Mental Health, University Health Network, Toronto, ON, Canada

Emily Choquette Department of Psychology, University of South Florida, Tampa, FL, USA

Anna C. Ciao Department of Psychology, Western Washington University, Bellingham, WA, USA

Laurence Claes Faculty of Psychology and Educational Sciences, KU Leuven, Leuven, Belgium

Faculty of Medicine and Health Sciences (CAPRI), University of Antwerp, Antwerp, Belgium

Simon Clarke Department of Adolescent Medicine, Westmead Hospital, Westmead, NSW, Australia

Centre for Research into Adolescent Health, Westmead Hospital, Westmead, NSW, Australia

Massimo Clerici Department of Medicine and Surgery, University of Milano-Bicocca, Milan, Italy

Department of Mental Health, San Gerardo Hospital, Monza, Italy

Fabrizia Colmegna Department of Mental Health, San Gerardo Hospital, Monza, Italy

Patricia Colton Department of Psychiatry, University of Toronto and Eating Disorders Program, University Health Network, Toronto, ON, Canada

Brian Cook Kinesiology Department, California State University Monterey Bay, Seaside, CA, USA

Lesley Cook Partners in Practice, Sydney, NSW, Australia

Myra Cooper Harris Manchester College, University of Oxford, Oxford, UK

Zafra Cooper Department of Psychiatry, Warneford Hospital, Oxford University, Oxford, UK

Samuele Cortese University of Southampton, Southampton, UK 
Elizabeth W. Cotter Department of Health Studies, American University, Washington, DC, USA

Jennifer Couturier Department of Psychiatry and Behavioural Neurosciences, McMaster University, Hamilton, ON, Canada

Ross D. Crosby Neuropsychiatric Research Institute, Fargo, ND, USA

University of North Dakota School of Medicine and Health Sciences, Fargo, ND, USA

Scott J. Crow Department of Psychiatry, Minnesota Obesity Center, University of Minnesota, Minneapolis, MN, USA

The Emily Program, Saint Paul, MN, USA

Antonios Dakanalis Department of Medicine and Surgery, University of Milano-Bicocca, Milan, Italy

Department of Brain and Behavioral Sciences, University of Pavia, Pavia, Italy

Riccardo Dalle Grave Department of Eating and Weight Disorders, Villa Garda Hospital, Garda (VR), Italy

Alison Darcy Stanford University, Palo Alto, CA, USA

Department of Psychiatry, Stanford University School of Medicine, Stanford, CA, USA

Lisa Dawson Department of Psychological Medicine, The Children's Hospital at Westmead, Westmead, NSW, Australia

Kathleen De Cuyper University Psychiatric Center, KU Leuven, Leuven, Belgium

Faculty of Psychology and Educational Sciences, KU Leuven, Leuven, Belgium

Jade DeWitt Department of Psychiatry, Columbia University Medical Center, New York, NY, USA

Ester Di Giacomo Department of Medicine and Surgery, University of Milano-Bicocca, Milan, Italy

Phillippa C. Diedrichs Centre for Appearance Research, University of the West of England, Bristol, UK

Sarah E. Domoff Center for Human Growth and Development, University of Michigan, Ann Arbor, MI, USA

Kyle P. De Young Department of Psychology, University of North Dakota, Grand Forks, ND, USA

David M. Dunkley Department of Psychiatry, Lady Davis Institute - Jewish General Hospital, Montreal, QC, Canada

Department of Psychiatry, McGill University, Montreal, QC, Canada

Department of Psychology, McGill University, Montreal, QC, Canada 
Patricia E. Dunne Department of Counseling and Clinical Psychology, Teachers College, Columbia University, New York, NY, USA

Nora Durkin Department of Psychiatry, University of Minnesota Medical School, Minneapolis, MN, USA

Kamryn T. Eddy Department of Psychiatry, Harvard Medical School, Boston, MA, USA

Eating Disorders Clinical and Research Program, Massachusetts General Hospital, Boston, MA, USA

Dawn M. Eichen University of California-San Diego, San Diego, CA, USA

Ivan Eisler Institute of Psychology, Psychiatry, and Neuroscience, King's College London, London, UK

Jo M. Ellison Neuropsychiatric Research Institute, Fargo, ND, USA

Scott G. Engel Neuropsychiatric Research Institute, Fargo, ND, USA

University of North Dakota School of Medicine and Health Sciences, Fargo, ND, USA

A. Kate Fairweather-Schmidt Flinders University, Adelaide, SA, Australia Christine Feinle-Bisset Discipline of Medicine, Royal Adelaide Hospital, University of Adelaide, Adelaide, SA, Australia

Fernando Fernández-Aranda Eating Disorders Unit, Department of Psychiatry, University Hospital of Bellvitge-IDIBELL, Barcelona, Spain

CIBER Fisiopatología de la Obesidad y la Nutrición (CIBERobn), Instituto Salud Carlos III, Madrid, Spain

Department of Clinical Sciences, School of Medicine, University of Barcelona, Barcelona, Spain

Marta Ferrer-Garcia Institute of Neurosciences, University of Barcelona, Barcelona, Spain

Manfred Maximilian Fichter Department of Psychiatry and Psychotherapy, Ludwig-Maximilians-University (LMU), Munich, Munich, Germany

Schön Klinik Roseneck affiliated with the Medical Faculty of the University of Munich (LMU), Prien, Germany

Ellen E. Fitzsimmons-Craft Weight Management and Eating Disorders Research Program, Department of Psychiatry, Washington University, St. Louis, MO, USA

Kelsie T. Forbush Department of Psychology, University of Kansas, Lawrence, KS, USA

Lauren N. Forrest Department of Psychology, Miami University, Oxford, $\mathrm{OH}, \mathrm{USA}$

Debra L. Franko Department of Applied Psychology, Northeastern University, Boston, MA, USA 
Hans-Christoph Friederich Department of Psychosomatic Medicine and Psychotherapy, LVR Klinikum, University Duisburg-Essen, Essen, Germany

Anthea Fursland Centre for Clinical Interventions, Perth, WA, Australia School of Psychology and Speech Pathology, Curtin University, Perth, WA, Australia

David M. Garner River Centre Clinic, River Centre Foundation, Sylvania, $\mathrm{OH}, \mathrm{USA}$

Jennifer L. Gaudiani ACUTE Center for Eating Disorders, Denver Health, Denver, CO, USA

University of Colorado School of Medicine, Denver, CO, USA

Diana L. Gaydusek American School of Professional Psychology, Department of Clinical Psychology, Argosy University, Washington, DC, Arlington, VA, USA

Josie Geller Eating Disorders Program, University of British Columbia, St. Paul's Hospital, Vancouver, BC, Canada

Ata Ghaderi Division of Psychology, Department of Clinical Neuroscience, Karolinska Institutet, Stockholm, Sweden

E. Leigh Gibson Department of Psychology, Whitelands College, University of Roehampton, London, UK

Deborah R. Glasofer Department of Psychiatry, Columbia University College of Physicians and Surgeons, New York, NY, USA

Division of Clinical Therapeutics, New York State Psychiatric Institute, New York, NY, USA

Eating Disorders Research Unit, New York State Psychiatric Institute/Columbia University Medical Center, New York, NY, USA

D. Glennon Eating Disorders Unit, South London and Maudsley NHS Foundation Trust, London, UK

Kate Godfrey Eating Disorder Service, The Children's Hospital at Westmead, NSW, Australia

Neville H. Golden Division of Adolescent Medicine, Department of Pediatrics, Lucile Packard Children's Hospital, Stanford University School of Medicine, Palo Alto, CA, USA

Rebecca J. Gomez Department of Psychiatry, University of Ottawa, Ottawa, Canada

Erica L. Goodman University of North Dakota, Grand Forks, ND, USA

Alexandria M. Goodyear Prevention Institute, Oakland, CA, USA

Catherine M. Gordon Division of Adolescent and Transition Medicine, Cincinnati Children's Hospital Medical Center, University of Cincinnati College of Medicine, Cincinnati, OH, USA 
Sasha Gorrell College of Arts and Sciences, Department of Psychology, University at Albany, State University of New York, Albany, NY, USA

Carlos M. Grilo Yale University School of Medicine, New Haven, CT, USA

Angela S. Guarda Department of Psychiatry and Behavioral Sciences, Johns Hopkins University School of Medicine, Baltimore, MD, USA

Anna I. Guerdjikova Lindner Center of HOPE, Mason, OH, USA

Department of Psychiatry and Behavioral Neuroscience, University of Cincinnati College of Medicine, Cincinnati, OH, USA

Gabriella Guzman Department of Psychiatry, Columbia Center for Eating Disorders, Columbia University Medical Center and New York State Psychiatric Institute, New York, NY, USA

Alissa A. Haedt-Matt Department of Psychology, Illinois Institute of Technology, Chicago, IL, USA

Andrea S. Hartmann Department of Human Sciences, Institute of Psychology, Osnabrück University, Osnabrück, Lower Saxony, Germany

Aaron Harwell Department of Psychology, Trinity University, San Antonio, TX, USA

Phillipa J. Hay School of Medicine, Western Sydney University, Sydney, NSW, Australia

School of Medicine, James Cook University, Townsville, QLD, Australia

Stephen N. Haynes Psychology Department, University of Hawai'i at Mānoa, Honolulu, HI, USA

Ann F. Haynos Department of Psychiatry, Duke University Medical Center, Durham, NC, USA

Department of Psychology, University of Nevada, Reno, NV, USA

Todd Heatherton University of Toronto Mississauga, Mississauga, ON, Canada

Roelie J. Hempel Psychology, University of Southampton, Southampton, UK

Prudence J. Hepple School of Psychology, The University of Western Australia, Crawley, WA, Australia

Beate Herpertz-Dahlmann Department of Child and Adolescent Psychiatry, Psychosomatics and Psychotherapy, University Clinics Technical University Aachen, Aachen, Germany

Marcia Herrin Dartmouth Geisel Medical School, Hanover, NH, USA

Gabriella Heruc Discipline of Medicine, Royal Adelaide Hospital, University of Adelaide, Adelaide, SA, Australia

Wolfgang Herzog Department of General Internal Medicine and Psychosomatics, Medical University Hospital Heidelberg, Heidelberg, Germany 
Anja Hilbert Behavioral Medicine, Integrated Research and Treatment Center Adiposity Diseases, University of Leipzig Medical Center, Leipzig, Saxony, Germany

Elizabeth Markley Holm Holm Nutrition Counseling, Alexandria, VA, USA

James I. Hudson McLean Hospital, Belmont, MA, USA

Department of Psychiatry, Harvard Medical School, Boston, MA, USA

Kathryn M. Huryk Fairleigh Dickinson University, Teaneck, NJ, USA

Corinna Jacobi Department of Clinical Psychology and Psychotherapy, Technische Universität Dresden, Dresden, Germany

Kristin N. Javaras McLean Hospital, Belmont, MA, USA

Department of Psychiatry, Harvard Medical School, Boston, MA, USA

Susana Jimenez-Murcia Eating Disorders Unit, Department of Psychiatry, University Hospital of Bellvitge-IDIBELL, Barcelona, Spain

CIBER Fisiopatología de la Obesidad y la Nutrición (CIBERobn), Instituto Salud Carlos III, Madrid, Spain

Department of Clinical Sciences, School of Medicine, University of Barcelona, Barcelona, Spain

Jennifer Jordan Department of Psychological Medicine, University of Otago, Christchurch, New Zealand

Canterbury District Health Board, Christchurch, New Zealand

Adrienne Juarascio Drexel University, Philadelphia, PA, USA

Melissa Kalarchian School of Nursing, Duquesne University, Pittsburgh, PA, USA

Anna M. Karam Department of Psychology, Washington University in St. Louis, St. Louis, MO, USA

Debra K. Katzman Division of Adolescent Medicine, Department of Pediatrics, The Hospital for Sick Children and University of Toronto, Toronto, ON, Canada

Undergraduate Medical Education, University of Toronto School of Medicine, Toronto, ON, Canada

Pamela K. Keel Department of Psychology, Florida State University, Tallahassee, FL, USA

Allison C. Kelly Department of Psychology, University of Waterloo, Waterloo, ON, Canada

Mackenzie C. Kelly Psychology Department, University of Hawai'i at Mānoa, Honolulu, HI, USA 
Nichole R. Kelly Department of Human Development and Family Studies, Section on Growth and Obesity, Program in Developmental Endocrinology and Genetics, Eunice Kennedy Shriver National Institute of Child Health and Human Development Colorado State University, Bethesda, MD, USA

Department of Medical and Clinical Psychology, Uniformed Services University of the Health Sciences, Bethesda, MD, USA

Kelly L. Klump Department of Psychology, Michigan State University, East Lansing, MI, USA

Lauren E. Knauf College of Arts and Sciences, Department of Psychology, University at Albany, State University of New York, Albany, NY, USA

Antonia Koskina Eating Disorders Unit, South London and Maudsley NHS Foundation Trust, London, UK

Helena Chmura Kraemer Department of Psychiatry and Behavioral Sciences, Stanford School of Medicine, Stanford University (Emerita), Stanford, CA, USA

Isabel Krug Melbourne School of Psychological Sciences, The University of Melbourne, Melbourne, VIC, Australia

Pei-Yoong Lam Division of Adolescent Medicine, Department of Pediatrics, University of British Columbia, Vancouver, BC, Canada

Provincial Specialized Eating Disorders Program, British Columbia Children's Hospital, Vancouver, BC, Canada

Amy M. Lampard Faculty of Health Sciences, School of Psychology and Speech Pathology, Curtin University, Bentley, WA, Australia

Katie Lang Department of Psychological Medicine, Institute of Psychiatry, Psychology and Neuroscience, King's College London, London, UK

Janet D. Latner Psychology Department, University of Hawai’i at Mānoa, Honolulu, HI, USA

Jason M. Lavender Neuropsychiatric Research Institute, Fargo, ND, USA University of North Dakota School of Medicine and Health Sciences, Fargo, ND, USA

Jocelyn Lebow Department of Psychiatry and Behavioral Sciences, University of Miami Miller School of Medicine, Miami, FL, USA

Department of Psychiatry and Psychology, Mayo Clinic College of Medicine, Rochester, MN, USA

Daniel Le Grange Department of Psychiatry, University of California, San Francisco, San Francisco, CA, USA

Monica Leslie King's College London, London, UK

Michael P. Levine Department of Psychology, Kenyon College, Gambier, $\mathrm{OH}$, USA 
Helena Lewis-Smith Centre for Appearance Research, University of the West of England, Bristol, UK

Lisa Rachelle Riso Lilenfeld Department of Clinical Psychology, American School of Professional Psychology at Argosy University, Washington DC, Arlington, VA, USA

Tanya Little Discipline of Medicine, Royal Adelaide Hospital, University of Adelaide, Adelaide, SA, Australia

James Lock Department of Psychiatry and Behavioral Science, Stanford University School of Medicine, Stanford, CA, USA

Katharine L. Loeb Fairleigh Dickinson University, Teaneck, NJ, USA

Katie A. Loth Department of Psychiatry, University of Minnesota Medical School - Twin Cities Campus, Minneapolis, MN, USA

Department of Family Medicine and Community Health, University of Minnesota Medical School, Minneapolis, MN, USA

Jennifer D. Lundgren Department of Psychology, University of MissouriKansas City, Kansas City, MO, USA

Xiaochen Luo Department of Psychology, Michigan State University, East Lansing, MI, USA

Janet A. Lydecker Department of Psychiatry, Yale School of Medicine, New Haven, CT, USA

Thomas R. Lynch Psychology, University of Southampton, Southampton, UK

Sloane Madden Sydney Medical School, Paediatrics and Child Health, Children's Hospital, University of Sydney, Westmead, NSW, Australia

Sarah Maguire Clinical Psychology and Psychiatry, University of Sydney, Centre for Eating and Dieting Disorders (CEDD), Sydney, NSW, Australia

Stephanie Manasse Drexel University, Philadelphia, PA, USA

Marcela A. Marin Dapelo Department of Psychological Medicine, Institute of Psychiatry, Psychology and Neuroscience, King's College London, London, UK

Emily Matheson Flinders University, Bedford Park, SA, Australia

Jessica McClelland Department of Psychological Medicine, Institute of Psychiatry, Psychology and Neuroscience, King's College London, London, UK

Una McCrann Department of Child and Adolescent Mental Health, Great Ormond Street Hospital for Children NHS Trust, London, UK

Susan L. McEIroy Lindner Center of HOPE, Mason, OH, USA

Department of Psychiatry and Behavioral Neuroscience, University of Cincinnati College of Medicine, Cincinnati, OH, USA 
Traci McFarlane University Health Network, Toronto General Hospital, Eating Disorder Program, Toronto, ON, Canada

Department of Psychiatry, University of Toronto, Toronto, ON, Canada

Virginia V. W. McIntosh Department of Psychological Medicine, University of Otago, Christchurch, New Zealand

Canterbury District Health Board, Christchurch, New Zealand

Siân A. McLean Department of Psychology and Counselling, School of Psychology and Public Health, La Trobe University, Melbourne, VIC, Australia

Sara McMullin Weight Management and Eating Disorders Program, Department of Psychiatry, Washington University, St. Louis, MO, USA

Gail L. McVey Ontario Community Outreach Program for Eating Disorders, University Health Network, Toronto, ON, Canada

Dalla Lana School of Public Health, University of Toronto, Toronto, ON, Canada

Katharine Menke Department of Psychology, University of Texas at Austin, Austin, TX, USA

Caroline Meyer WMG and Warwick Medical School, University Hospitals Coventry and Warwickshire NHS Trust, University of Warwick, Coventry, UK

Nadia Micali Institute of Child Health, University College London, London, UK

Department of Psychiatry, Icahn School of Medicine at Mount Sinai, New York, NY, USA

James E. Mitchell Department of Clinical Research, Neuropsychiatric Research Institute, Fargo, ND, USA

Department of Psychiatry and Behavioral Science, University of North Dakota School of Medicine and Health Sciences, Fargo, ND, USA

Jessica Moncrieff-Boyd School of Psychology, University of Western Australia, Perth, Crawley, WA, Australia

Jonathan M. Mond Research School of Psychology, Australian National University, Canberra, Australia

Department of Psychology, Macquarie University, Sydney, NSW, Australia

Grace E. Monterubio Weight Management and Eating Disorders Research Program, Department of Psychiatry, Washington University, St. Louis, MO, USA

Christine Morgan The Butterfly Foundation, Melbourne, VIC, Australia

Molly Moroz Department of Psychiatry, Lady Davis Institute - Jewish General Hospital, Montreal, QC, Canada

Department of Psychology, McGill University, Montreal, QC, Canada 
Victoria A. Mountford Eating Disorders Unit, South London and Maudsley NHS Foundation Trust, London, UK

Eating Disorders Unit, Institute of Psychiatry, Psychology and Neuroscience, King's College London, London, UK

Melissa A. Munn-Chernoff Department of Psychiatry, University of North Carolina at Chapel Hill, Chapel Hill, NC, USA

Bethany R. Munson Department of Psychology, Western Washington University, Bellingham, WA, USA

Rebecca Murphy Department of Psychiatry, Oxford University, Warneford Hospital, Oxford, UK

Stuart B. Murray Department of Psychiatry, University of California, San Francisco, San Francisco, CA, USA

Helen Burton Murray Drexel University, Philadelphia, PA, USA

Jason M. Nagata Department of Pediatrics, Lucile Packard Children's Hospital, Stanford University School of Medicine, Palo Alto, CA, USA

Tom Nehmy School of Psychology, Flinders University, Bedford Park, SA, Australia

Dasha Nicholls Department of Child and Adolescent Mental Health, Great Ormond Street Hospital for Children NHS Trust, London, UK

UCL Great Ormond Street Institute of Child Health, London, UK

Greta Noordenbos Department of Clinical Psychology, Leiden University, Leiden, The Netherlands

Mark L. Norris Department of Pediatrics, Children's Hospital of Eastern Ontario, Ottawa, Canada

Erika Nyman-Carlsson Faculty of Medical Sciences, Örebro University, Örebro, Sweden

Capio Eating Disorder Centre, Stockholm, Sweden

Shannon M. O'Connor Department of Psychology, Michigan State University, East Lansing, MI, USA

Anne O'Shea School of Psychology, Flinders University, Adelaide, SA, Australia

J. O'Toole Kartini Clinic, Portland, OR, USA

Vartouhi Ohanian Schema Therapy UK, London, UK

Olivia C. Ohls Department of Psychology, Western Washington University, Bellingham, WA, USA

Marion Olmsted Department of Psychiatry, University of Toronto and Eating Disorders Program, University Health Network, Toronto, ON, Canada 
Susan J. Paxton Department of Psychology and Counselling, School of Psychology and Public Health, La Trobe University, Melbourne, VIC, Australia

Mia L. Pellizzer Faculty of Social and Behavioural Sciences, School of Psychology, Flinders University, Adelaide, SA, Australia

Jamie-Lee Pennesi School of Psychology, Flinders University, Adelaide, SA, Australia

Carol B. Peterson Department of Psychiatry, The University of Minnesota, Minneapolis, MN, USA

Kathleen M. Pike Department of Epidemiology, Mailman School of Public Health, Columbia University, New York, NY, USA

Department of Psychiatry, College of Physicians and Surgeons, Columbia University, New York, NY, USA

Abigail Pine Department of Medical and Clinical Psychology, Uniformed Services University of the Health Sciences (USUHS), Bethesda, MD, USA

Emily M. Pisetsky Department of Psychiatry, The University of Minnesota, Minneapolis, MN, USA

Maribel Plasencia Department of Psychology, Rutgers, The State University of New Jersey, Piscataway, NJ, USA

Carolyn Plateau National Centre for Sport and Exercise Medicine, Loughborough University, Leicestershire, UK

Franziska Plessow Department of Medicine, Harvard Medical School, Boston, MA, USA

Neuroendocrine Unit, Massachusetts General Hospital, Boston, MA, USA

Janet Polivy University of Toronto Mississauga, Mississauga, ON, Canada

Tarryn Pollard Department of Psychology, Trinity University, San Antonio, TX, USA

Pennington Biomedical Research Center, Baton Rouge, LA, USA

Ashleigh Pona Department of Psychology, University of Missouri-Kansas City, Kansas City, MO, USA

Norbert Quadflieg Department of Psychiatry and Psychotherapy, LudwigMaximilians-University (LMU), Munich, Munich, Germany

Deborah Lynn Reas Regional Department of Eating Disorders (RASP), Division of Mental Health and Addiction, Oslo University Hospital, Oslo, Norway

Graham W. Redgrave Department of Psychiatry and Behavioral Sciences, Johns Hopkins University School of Medicine, Baltimore, MD, USA

Erin E. Reilly College of Arts and Sciences, Department of Psychology, University at Albany, State University of New York, Albany, NY, USA 
Rachel Ress Department of Medical and Clinical Psychology, Uniformed Services University of the Health Sciences (USUHS), Bethesda, MD, USA

Lina A. Ricciardelli School of Psychology, Faculty of Health, Deakin University, Burwood, VIC, Australia

Jonathan H. Richardson Charis Center for Eating Disorders, Indiana University Health/Department of Pediatrics, Indiana University School of Medicine, Indianapolis, IN, USA

Sharon Ridley Department of Health in Western Australia, Centre for Clinical Interventions, Perth, WA, Australia

Danielle Ridolfi Weight Management and Eating Disorders Program, Department of Psychiatry, Washington University, St. Louis, MO, USA

Melissa Riegel Division of Clinical Therapeutics, New York State Psychiatric Institute, New York, NY, USA

Elizabeth Rieger Research School of Psychology, The Australian National University, Canberra, ACT, Australia

Giuseppe Riva Department of Psychology, Catholic University of Milan, Milan, Italy

Applied Technology for Neuro-Psychology Laboratory, IRCSS Istituto Auxologico Italiano, Milan, Italy

Tomi-Ann Roberts Department of Psychology, Colorado College, Colorado Springs, CO, USA

Annaleise Robertson Eating Disorder Service, The Children's Hospital at Westmead, NSW, Australia

Lauren Robinson Institute of Child Health, University College London, London, UK

Rachel F. Rodgers Department of Applied Psychology, Northeastern University, Boston, MA, USA

Laboratoire de Stress Traumatique, Universite Paul Sabatier, Toulouse, France

Vanja Rozenblat Melbourne School of Psychological Sciences, The University of Melbourne, Melbourne, VIC, Australia

Janice Russell Discipline of Psychiatry, Sydney Medical School, University of Sydney, Sydney, NSW, Australia

Debra L. Safer Department of Psychiatry and Behavioral Sciences, Stanford University School of Medicine, Stanford, CA, USA

Lauren E. Salci Department of Psychology and Counselling, School of Psychology and Public Health, La Trobe University, Melbourne, VIC, Australia 
Ulrike Schmidt Institute of Psychiatry, Psychology and Neuroscience, King's College London, London, UK

Natasha A. Schvey Department of Medical and Clinical Psychology, Uniformed Services University of the Health Sciences (USUHS), Bethesda, MD, USA

Ia Shekriladze Dimitri Uznadze Institute of Psychology, Ilia State University, Tbilisi, Georgia

April R. Smith Department of Psychology, Miami University, Oxford, OH, USA

Meghan L. Smith Department of Epidemiology, Mailman School of Public Health, Columbia University, New York, NY, USA

Wendy Spettigue Department of Psychiatry, Children's Hospital of Eastern Ontario, Ottawa, Canada

Claire J. Starrs Department of Psychiatry, Lady Davis Institute - Jewish General Hospital, Montreal, QC, Canada

Department of Psychiatry, McGill University, Montreal, QC, Canada

Howard Steiger Eating Disorders Program, Douglas Institute, Montréal, QC, Canada

Psychiatry Department, McGill University, Montréal, QC, Canada

Joanna E. Steinglass Department of Psychiatry, Columbia University Medical Center and New York State Psychiatric Institute, New York, NY, USA

Trevor Steward Eating Disorders Unit, Department of Psychiatry, University Hospital of Bellvitge-IDIBELL, Barcelona, Spain

CIBER Fisiopatología de la Obesidad y la Nutrición (CIBERobn), Instituto Salud Carlos III, Madrid, Spain

Tiffany M. Stewart Pennington Biomedical Research Center, Baton Rouge, LA, USA

Eric Stice Department of Psychology, University of Texas at Austin, Austin, TX, USA

Clinical Psychology, Oregon Research Institute, Eugene, OR, USA

Robyn Sysko Eating and Weight Disorders Program, Icahn School of Medicine at Mount Sinai, New York, NY, USA

Amy Talbot Dr Amy Talbot and Associates, Sydney, NSW, Australia

Marian Tanofsky-Kraff Department of Medical and Clinical Psychology, Uniformed Services University of the Health Sciences (USUHS), Bethesda, MD, USA 
Madeleine Tatham Norfolk Community Eating Disorders Service, Cambridge Partnership NHS Trust, London, UK

C. Barr Taylor Department of Psychiatry and Behavioral Sciences, Stanford University School of Medicine, Stanford, CA, USA

Kate Tchanturia Department of Psychological Medicine, Institute of Psychiatry, Psychology and Neuroscience, King's College London, London, UK Ilia State University, Tbilisi, Georgia

Michel A. Thibodeau Eating Disorder Program Toronto General Hospital University Health Network, Toronto, ON, Canada

Jennifer J. Thomas Eating Disorders Clinical and Research Program, Massachusetts General Hospital, Boston, MA, USA

Department of Psychiatry, Harvard Medical School, Boston, MA, USA

Stephanie Thomas Departments of Psychiatry, University of North Carolina at Chapel Hill, Chapel Hill, NC, USA

J. Kevin Thompson Department of Psychology, University of South Florida, Tampa, FL, USA

Chris Thornton The Redleaf Practice, Wahroonga, NSW, Australia

Stephen Touyz Clinical Psychology and Psychiatry, University of Sydney, Centre for Eating and Dieting Disorders (CEDD), Sydney, NSW, Australia

Katherine Travis Department of Pediatrics, Stanford School of Medicine, Stanford, CA, USA

Janet Treasure Psychological Medicine, King's College London, IOPPN, London, UK

Kathryn Trottier University Health Network, Toronto General Hospital, Eating Disorder Program, Toronto, ON, Canada

Department of Psychiatry, University of Toronto, Toronto, ON, Canada

Hannah Turner Eating Disorders Service, Southern Health NHS Foundation Trust, and Psychology Academic Unit, University of Southampton, Southampton, UK

Linsey M. Utzinger Department of Clinical Research, Neuropsychiatric Research Institute, Fargo, ND, USA

Department of Psychiatry and Behavioral Science, University of North Dakota School of Medicine and Health Sciences, Fargo, ND, USA

Eva Vall Faculty of Social and Behavioural Sciences, School of Psychology, Flinders University, Adelaide, SA, Australia

Tatjana van Strien Department of Earth and Life sciences, Free University, Amsterdam, Netherlands

Behavioural Science Institute, Radboud University Nijmegen, Nijmegen, Netherlands 
Johan Vanderlinden University Psychiatric Center, KU Leuven, Leuven, Belgium

Faculty of Psychology and Educational Sciences, KU Leuven, Leuven, Belgium

Jessica L. VanHuysse Genesys Regional Medical Center/MSU College of Human Medicine, East Lansing, MI, USA

Christina Verzijl Department of Psychology, Trinity University, San Antonio, TX, USA

Kristin M. von Ranson Department of Psychology, University of Calgary, Calgary, AB, Canada

Stamatoula Voulgari South London and Maudsley NHS Foundation Trust, London, UK

Elske Vrieze University Psychiatric Center, KU Leuven, Leuven, Belgium

Tracey Wade School of Psychology, Flinders University, Adelaide, SA, Australia

Deborah Waller NHS GP Surgery, Oxford, UK

Glenn Waller Clinical Psychology Unit, Department of Psychology, The University of Sheffield, Sheffield, South Yorkshire, UK

Andrew Wallis Eating Disorder Service, The Children's Hospital at Westmead, NSW, Australia

B. Timothy Walsh Eating Disorders Research Unit, New York State Psychiatric Institute/Columbia University Medical Center, New York, NY, USA

Warren Ward School of Medicine, University of Queensland, Brisbane, QLD, Australia

Heather Westwood King's College London, London, UK

Amy Wicksteed Sheffield Eating Disorders Service, Sheffield Health and Social Care NHS Foundation Trust, South Yorkshire, UK

Jennifer E. Wildes Eating Disorders Program, Department of Psychiatry and Behavioral Neuroscience, The University of Chicago, Chicago, IL, USA

Denise E. Wilfley Weight Management and Eating Disorders Research Program, Department of Psychiatry, Washington University in St. Louis, St. Louis, MO, USA

Department of Psychiatry, Washington University in St. Louis, School of Medicine, St. Louis, MO, USA

Simon Wilksch School of Psychology, Flinders University, Adelaide, SA, Australia 
Stephen A. Wonderlich Department of Clinical Research, Neuropsychiatric Research Institute, Fargo, ND, USA

Department of Psychiatry and Behavioral Science, University of North Dakota School of Medicine and Health Sciences, Fargo, ND, USA

Almut Zeeck Department of Psychosomatic Medicine and Psychotherapy, Medical University Hospital Freiburg, Freiburg, Germany

Stephan Zipfel Department of Psychosomatic Medicine and Psychotherapy, Medical University Hospital Tübingen, Tübingen, Germany

Nancy Zucker Psychiatry and Behavioral Sciences, Duke University School of Medicine, Durham, NC, USA 